\title{
Assessing the Capability of Doppler Global Velocimetry To Measure Vortical Flow Fields
}

James F. Meyers

Jimmy W. Usry

NASA - Langley Research Center

Hampton, Virginia 23665

and

L. Scott Miller

Department of Aerospace Engineering

Wichita State University

Wichita, Kansas 67208

Journal of Aerospace Engineering part G, vol 208, pp. 99-105

December 1994 



\title{
Assessing the Capability of Doppler Global Velocimetry To Measure Vortical Flow Fields
}

\author{
by \\ James F. Meyers \\ Jimmy W. Usry \\ NASA - Langley Research Center \\ Hampton, Virginia 23665 \\ and \\ L. Scott Miller \\ Department of Aerospace Engineering \\ Wichita State University \\ Wichita, Kansas 67208
}

\begin{abstract}
SYNOPSIS A new nonintrusive flow diagnostics instrumentation system, Doppler global velocimetry, is presented. The system is capable of making simultaneous, three-component velocity measurements within a selected measurement plane at video camera rates. These velocity images can provide the researcher with spatial and temporal information about the flow field in a global sense. The investigation of a vortical flow above a 75-degree delta wing comparing standard threecomponent, fringe-type laser velocimetry measurements with Doppler global velocimetry measurements is presented.
\end{abstract}

\section{NOTATION}

$\begin{array}{ll}\mathrm{c} & \text { Speed of light, } \mathrm{m} / \mathrm{sec} \\ \hat{\imath} & \text { Laser beam propagation direction } \\ \text { IVC } & \text { Iodine vapor cell } \\ \hat{\mathrm{o}} & \text { Collected scattered light direction } \\ \mathrm{V} & \text { Velocity of a particle passing through the laser beam, } \mathrm{m} / \mathrm{sec} \\ X & \text { Cross tunnel coordinate, } \mathrm{m} \\ Y & \text { Vertical coordinate, } \mathrm{m} \\ Z & \text { Streamwise coordinate, } \mathrm{m} \\ \Delta v & \text { Doppler shift frequency, Hz }\end{array}$




$\begin{array}{ll}v & \text { Laser output frequency, } \mathrm{Hz} \\ \theta & \begin{array}{l}\text { Angle between the laser propagation direction and the } \\ \text { collected scattered light, deg }\end{array}\end{array}$

\section{INTRODUCTION}

The requirements of enhanced maneuverability and increased lift placed on modern aircraft designs call for a greater understanding of three-dimensional flow fields and their interaction with the designed aircraft. Presently this understanding is developed through a combination of theoretical predictions using computational fluid dynamics (CFD), experimental measurement of flows about wind tunnel models, and aircraft response during flight testing. While advances in CFD are providing three-dimensional information on dense planar grids, experimental investigations of these planes rely on sequential measurement techniques which require excessive wind tunnel time to obtain a fraction of the information provided by CFD.

In an effort to expand and improve experimental databases, NASA has initiated the development of a new nonintrusive global measurement technique, Doppler global velocimetry. This technique, invented by Komine et al. (1), can yield global, simultaneous, three-dimensional velocity measurements of the flow field within a selected measurement plane. These global velocity maps can be acquired at video camera rates from which mean velocity components, and velocity time histories can be extracted with spatial densities equivalent to those obtained with CFD.

In this paper, a prototype Doppler global velocimetry instrument system is described. Additionally, results of an investigation of the vortical flow above a 75-degree delta wing, conducted in the Basic Aerodynamics Research Tunnel, are assessed to determine the potential of the technique. A vortical flow was selected for investigation since this flow category is receiving increased application on high performance aircraft, and the results could be compared with previous fringe-type laser velocimetry measurements obtained under the same conditions. These tests are part of an ongoing research program to develop and refine Doppler global velocimetry for wind tunnel and flight applications.

\section{DOPPLER GLOBAL VELOCIMETRY}

The operating principle governing the Doppler global velocimeter, DGV, is the shift in optical frequency of scattered light from objects passing 
through a laser beam. This principle was exploited by Yeh and Cummins in 1964 (2) to develop the first reference-beam, laser Doppler velocimeter (LDV). As depicted in Fig 1, scattered light collected by a detector located along the direction ô, from particles passing through a laser beam propagating in direction $\hat{1}$, is Doppler shifted based on a velocity in the direction ( $\hat{o}-\hat{1})$. This relationship is expressed by:

$$
\Delta \mathrm{v}=\frac{\mathrm{v}_{o}(\hat{\mathrm{o}}-\hat{\mathrm{1}}) \cdot \mathrm{V}}{\mathrm{c}}
$$

where $\Delta v$ is the Doppler shifted frequency, $v$ is the laser frequency, $V$ is the particle velocity, and $c$ is the speed of light.

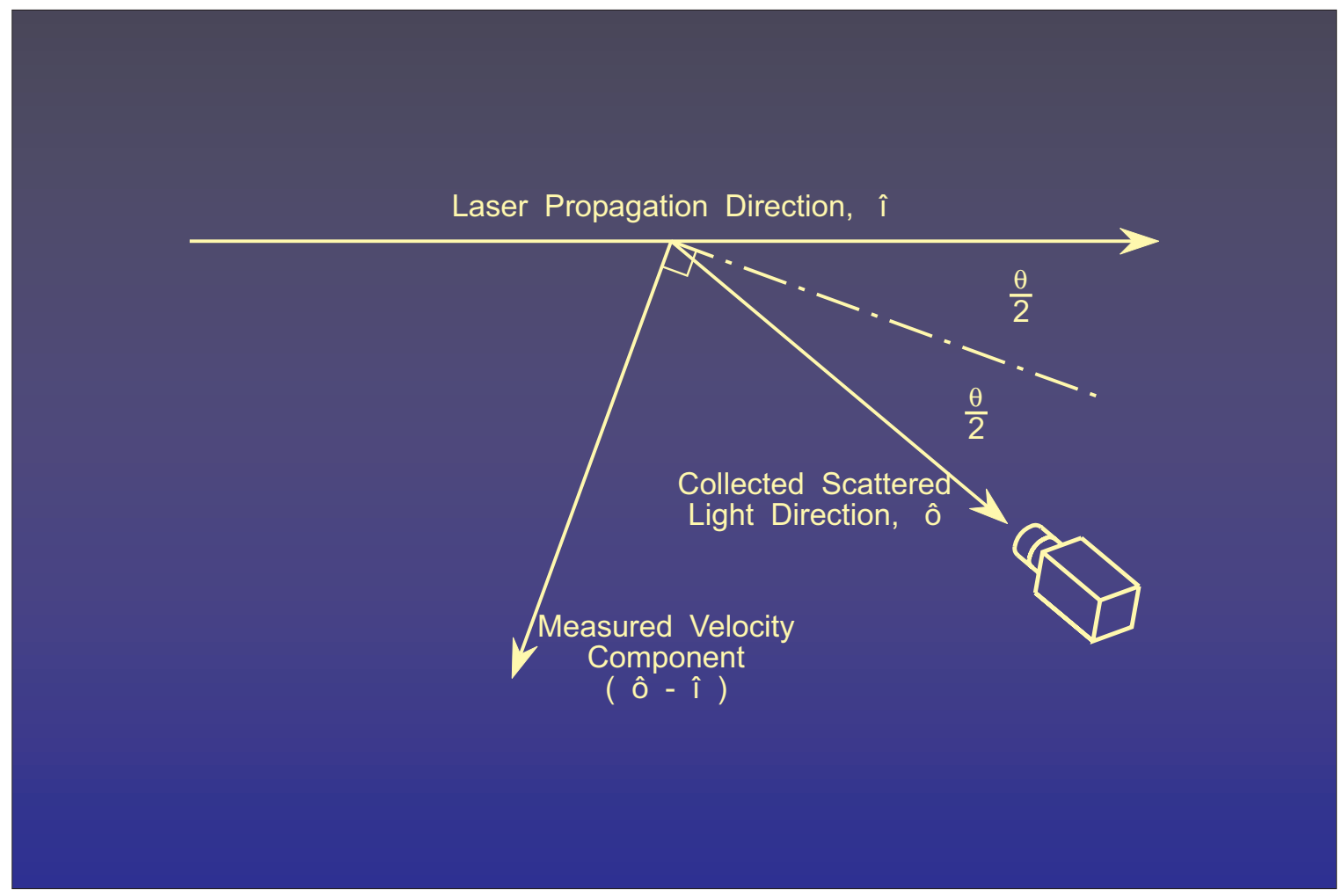

Fig 1. Diagram depicting the velocity measurement direction based on the orientation of the laser propagation direction and the detector location.

Whereas the LDV used optical heterodyning techniques to obtain the Doppler frequency, the DGV measures the optical frequency directly using an absorption line filter, (Iodine vapor cell), as a frequency discriminator. An Argon ion laser operating in single-line mode at $514.5 \mathrm{~nm}$ is tuned by adjusting the intercavity etalon to an optical frequency corresponding to a point midway along the edge of an Iodine absorption line, Fig 2. Collected scattered light from a stationary object or cloud of particles will be attenuated by 50 percent as it passes through 
the Iodine vapor. If the object or particle cloud is moving, the attenuation through the vapor will increase (or decrease, depending on the direction of movement) by an amount proportional to the Doppler shift. Since the Absorption Line Filter (ALF) measures light frequency directly, it is not restricted to scattered light from a single particle collected by a single detector as in the LDV. Thus if the laser beam is expanded into a light sheet and the detector replaced by a CCD camera, global velocity measurements can be obtained. A pictorial view of this method is shown in Fig 3. The function of the reference camera shown in Fig 3 is to obtain an intensity map from the illuminated particle field without the influence of the Doppler effect. This map is used to normalize the signal camera output and thus remove intensity variations caused by effects other than velocity, e.g., variations in particle number density, particle size, and laser power density. Further details on the operation of the DGV are given by Komine et al (1), Meyers and Komine (3), and Meyers et al (4).

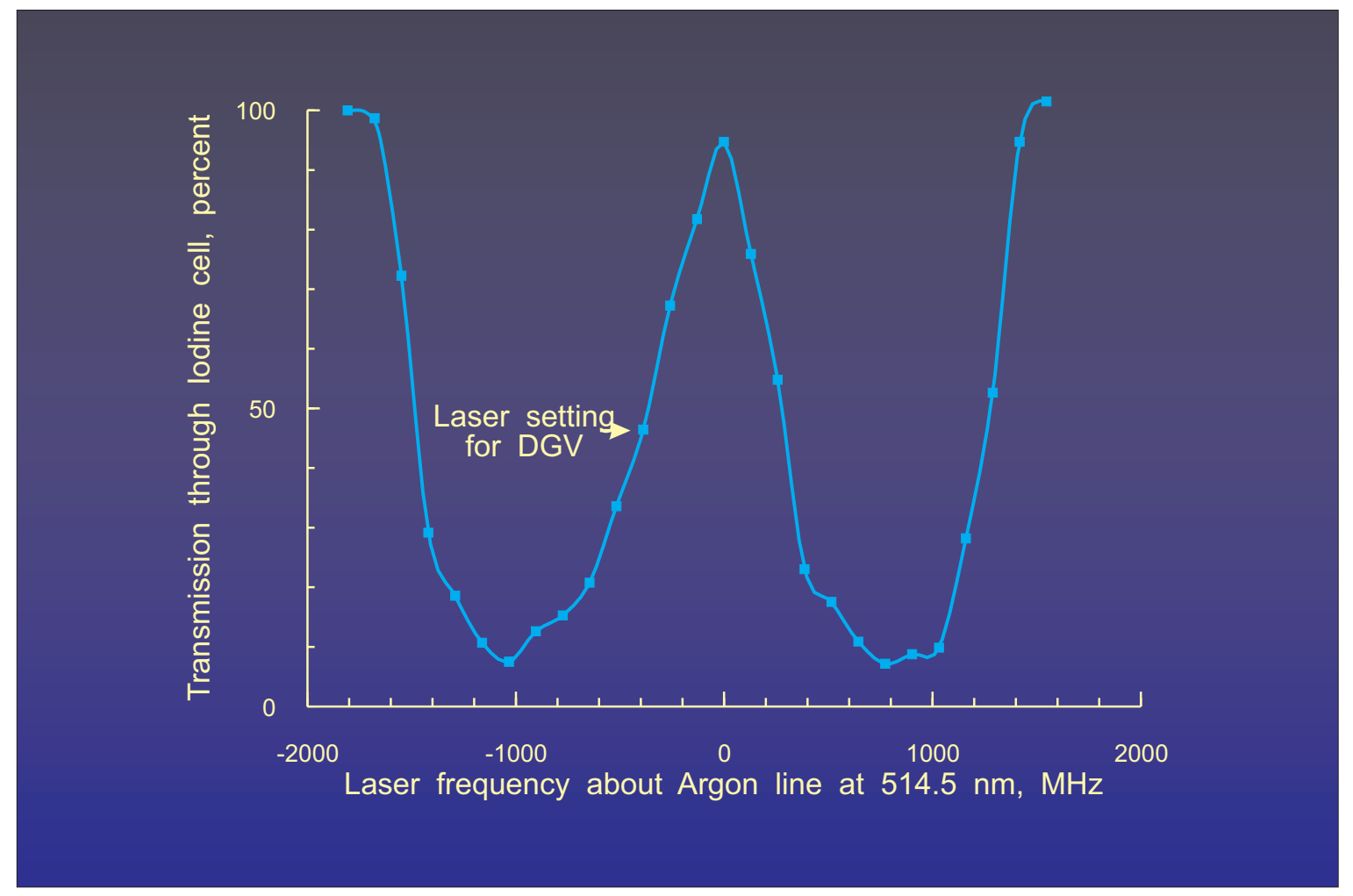

Fig 2. Transfer function of the Iodine vapor cell, IVC.

As stated above and depicted in Fig 1, the configuration measures the velocity component in the direction ( $\hat{o}-\hat{1})$. Moving the detector, thus changing the direction of ( $\hat{o}-\hat{1})$, allows another velocity componen to be measured. Likewise, changing the laser beam propagation direction, $\hat{\imath}$, will also change the direction of the measured velocity component. 
Therefore, a three-component DGV can be constructed by using multiple detectors and/or multiple laser beam propagation directions.

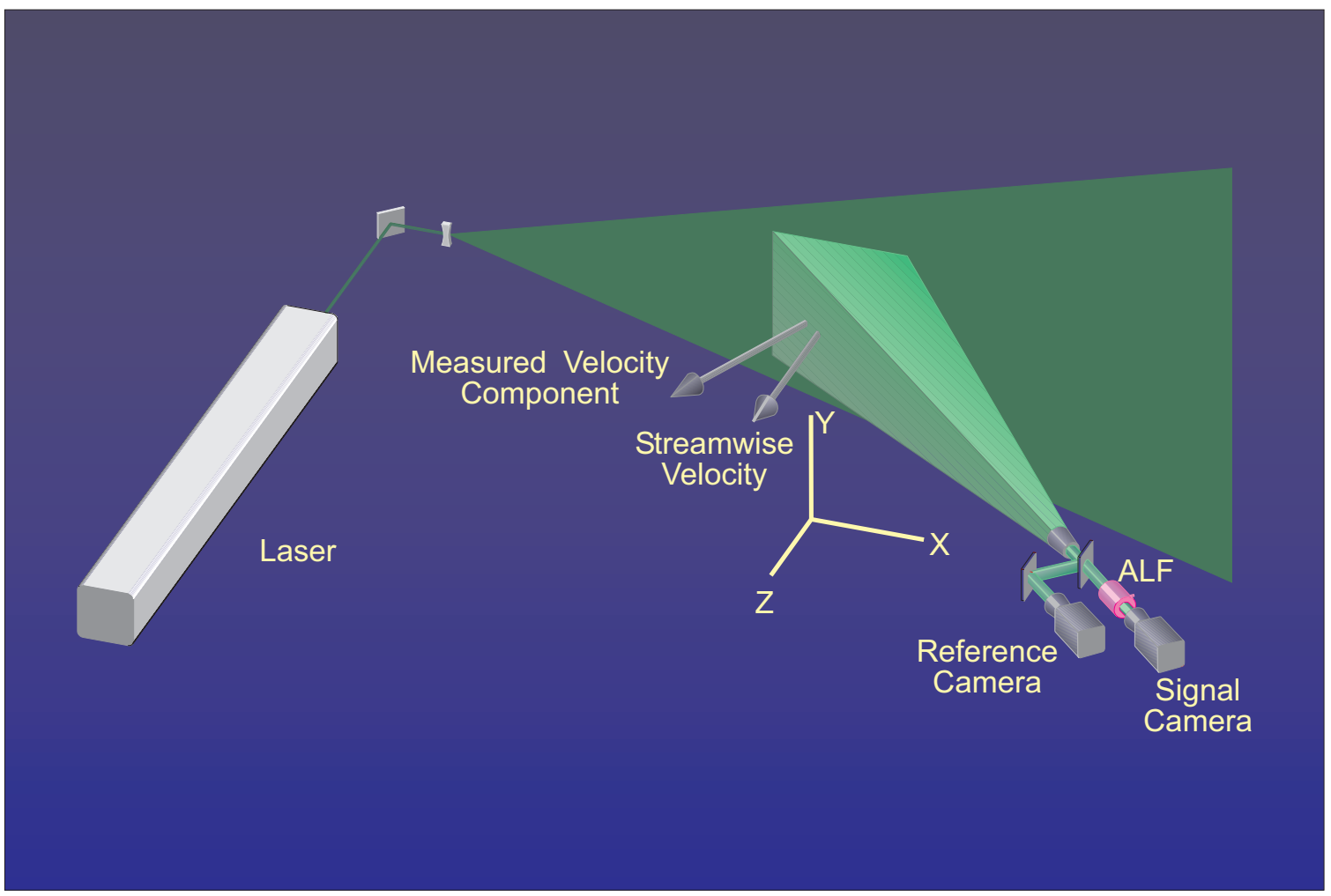

Fig 3. Pictorial view of the Doppler global velocimeter used in the Basic Aerodynamics Research Tunnel.

\section{MEASUREMENT OF THE CIRCULAR VELOCITY IN THE VORTICAL FLOW ABOVE A 75-DEGREE DELTA WING}

The potential of the Doppler global velocimeter was investigated by Usry et al (5) by measuring the vortical flow field above a 75-degree delta wing in the Basic Aerodynamic Research Tunnel. The facility, as described by Sellers and Kjelgaard (6), was a small, open return tunnel with a maximum velocity of $67 \mathrm{~m} / \mathrm{sec}$ with a Reynolds number per meter of 0.43 million. The airflow entering the tunnel was conditioned by a honeycomb structure, four antiturbulence screens, and an 11:1 contraction ratio. The free stream turbulence intensity was less than 0.08 percent for all flow conditions. The propylene glycol vaporization/condensation generator developed for vapor screen flow visualization was used as the source of particles for the experiment. The particles, injected at the inlet of the tunnel, had a size distribution, as measured by Meyers (7), which peaks at $0.7 \mu \mathrm{m}$ with a skewed distribution to a maximum of $10 \mu \mathrm{m}$. 
An Argon ion laser operating in $\mathrm{TEM}_{\mathrm{oo}}$ mode with an etalon to main tain single longitudinal mode at $514.5 \mathrm{~nm}$ was used as the light source. The output beam was directed to one of three cylindrical lenses to form a light sheet with the desired propagation directions $\left(\hat{\imath}_{1}, \hat{\imath}_{2}\right.$ and $\left.\hat{1}_{3}\right)$ to facilitate the measurement of three velocity components. The receiver optical system consisting of the collecting lens, beam splitter, IVC, and CCD cameras was located 53 degrees from the streamwise (tunnel centerline) direction in the horizontal plane, Fig 3. A photograph of the receiver optical system, viewing particles in the vortical flow passing through the light sheet above the delta wing, is shown in Fig 4 . The outputs from the reference and signal cameras were processed by both an analog normalization circuit and a digital dual frame grabber as described by Meyers et al (4). The analog processor consisted of a divider circuit that normalized the signal camera output by the reference camera signal in real time. The resulting signal was converted to a standard RS -170 video signal that was stored, along with the two camera signals, on a 450-line video disk recorder. The digital dual frame grabber simultaneously acquired a video frame from both cameras. The amplitudes of the measured light intensities for a given pixel in each frame were used to address a lookup table element containing the precomputed normalization value for those amplitudes. The resulting normalized image was passed, along with the original frames, to a microcomputer for further processing and storage.

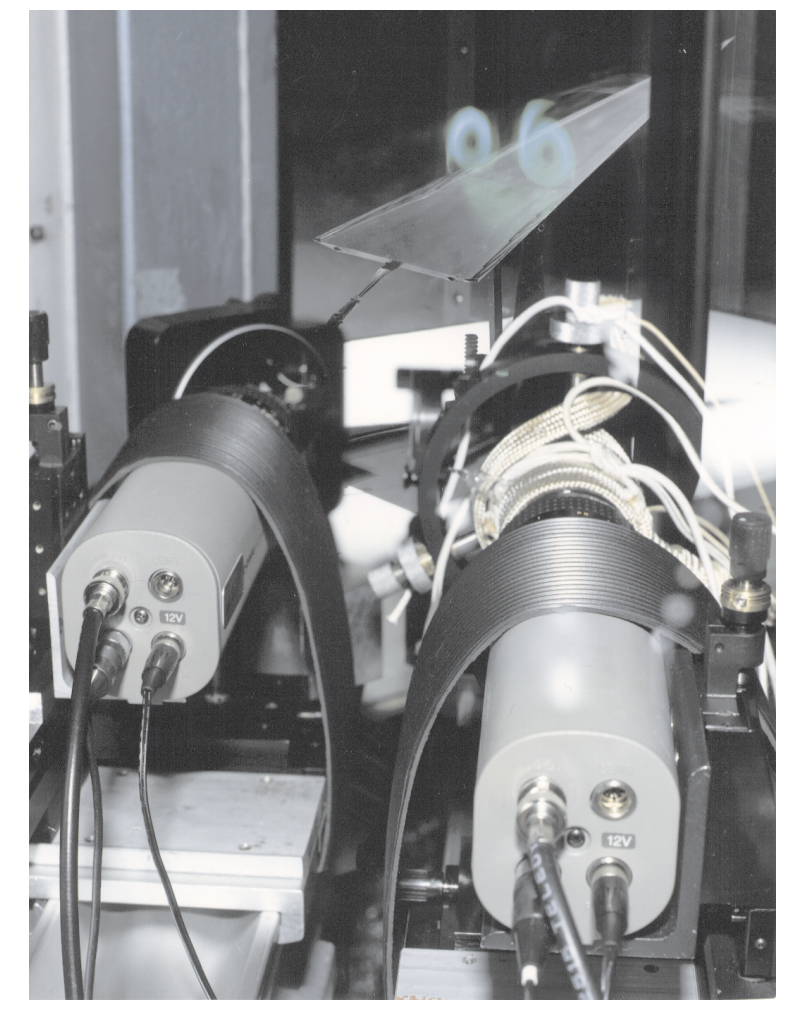

Fig 4. DGV receiver optical system installed in the Basic Aerodynamic Research Tunnel. 
A stainless steel 75-degree delta wing, $0.57 \mathrm{~m}$ in length, with sharp leading edges was placed in the tunnel at an angle of attack of 20.5 degrees. The tunnel dynamic pressure was set to $402 \mathrm{~N} / \mathrm{m}^{2}$ which yielded a freestream velocity of $40 \mathrm{~m} / \mathrm{sec}$. The laser light sheet was placed perpendicular to the tunnel centerline at the 70 percent chord location on the model. These conditions matched the model and tunnel settings used to acquire three component fringe-type, laser velocimeter measurements in the investigation presented by Sellers and Kjelgaard (6) and Meyers and Hepner (8). The results of the previous study will be used as the standard for comparison with the present DGV measurements. The laser velocimeter, LV, measurements obtained at the 70 percent chord location are shown in Fig 5 . The measurements

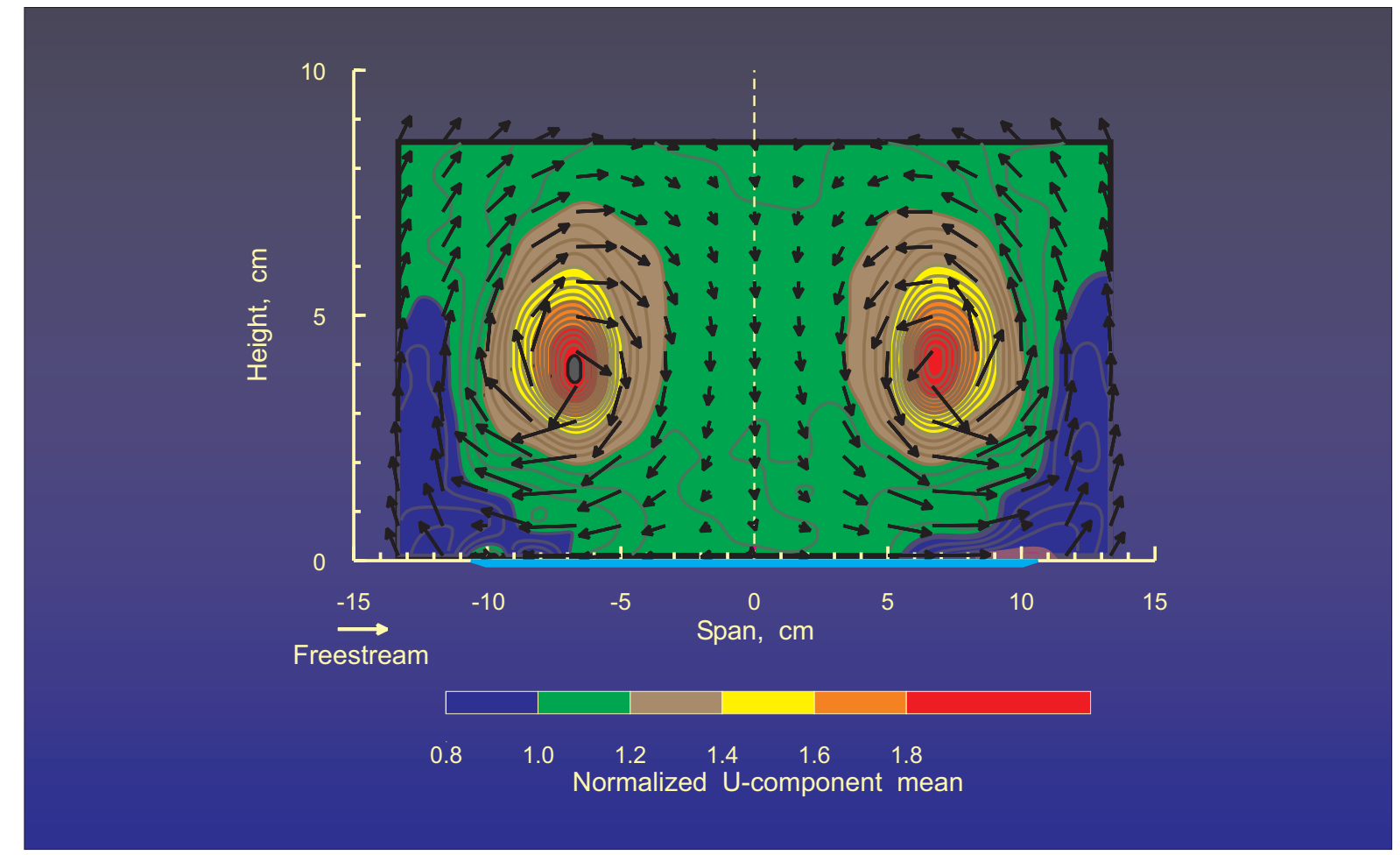

Fig 5. Three component laser velocimeter measurements of the vortical flow field above a 75 -degree delta wing at an angle-of-attack of 20.5 degrees.

obtained at 273 location s during an eight hour period of data acquisition were spline fit to yield the gray scale contours of streamwise velocity. The other two components were combined to produce arrows representing the velocity vector of the circular flow within the plane perpendicular to the streamwise direction. Note that the streamwise velocity accelerated to twice the freestream value at the vortex core. The circular flow was compressed by the wing and accelerated to 1.5 times the freestream value as the flow expanded outward below the 
core. Of the three measurement directions established by the DGV geometry, the direction that best illustrated the circular flow characteristics of the vortex was the nearly cross flow component, 71.5 degrees from the streamwise direction in the horizontal plane, Fig 6 . Resolving the three component LV data to obtain the velocity field along this direction yielded the contour map shown in Fig 7 . The average of 30 frames of DGV data (equivalent to 1 second of data acquisition) obtained by the digital dual frame grabber of the velocity field along this component is shown in Fig 8. Each pixel within this normalized image was gray-scale coded based on the measured velocity.

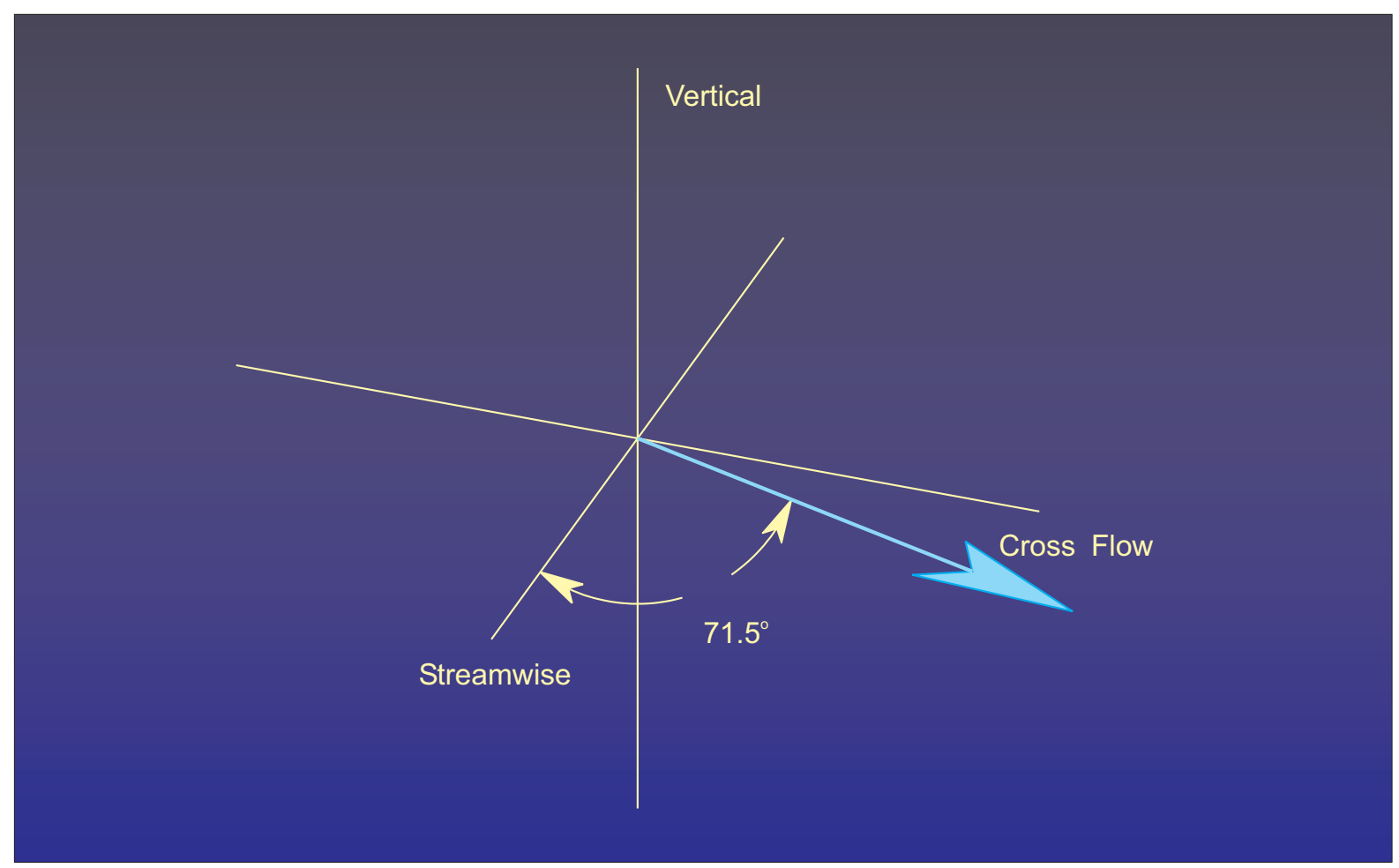

Fig 6. Measurement direction for DGV operation inbackscatter mode. 

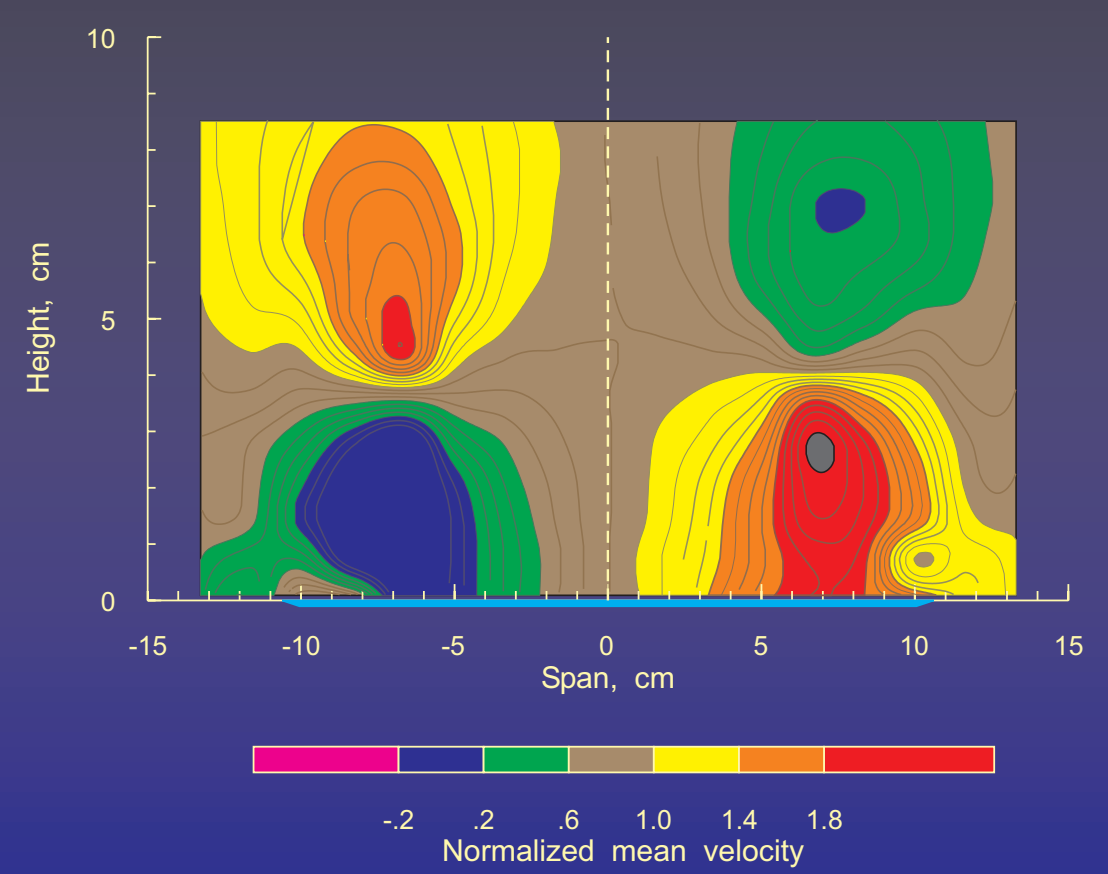

Fig 7. Resolved laser velocimeter measurements along the direction 71.5 degrees from streamwise in the horizontal plane.
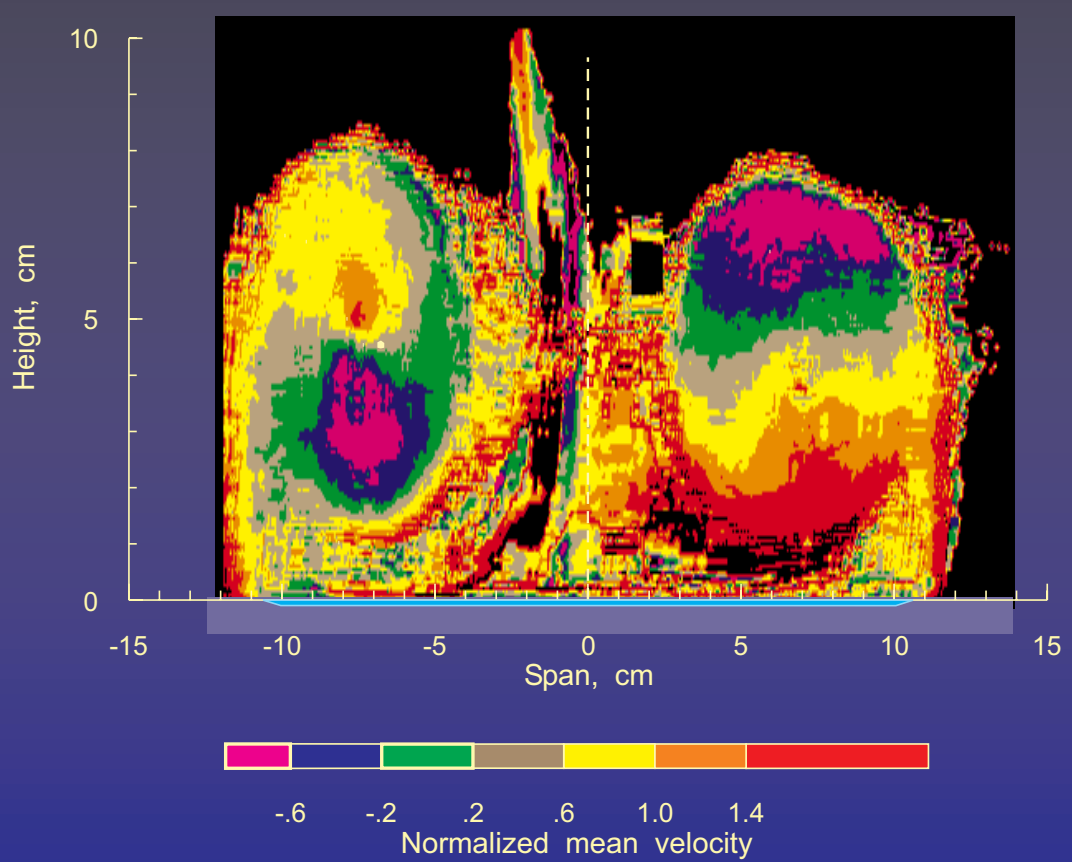

Fig 8. DGV measurements of the velocity field (average of 30 frames) for the component along the direction 71.5 degrees from streamwise in the horizontal plane. 
The straight-forward data processing scheme implemented within the digital dual frame grabber was performed with the assumption that pixel-by-pixel alignment between the two cameras was perfect. Unfortunately, the experiences during testing in the BART and the posttesting analysis of the data indicated that perfect alignment could not be obtained. Detailed laboratory investigations of the receiver optical system following the wind tunnel investigation found several problems preventing the exact overlay of the two images. These problems ranged from mismatched depths of field, and optical distortions caused by lens aberrations, imperfections in the beamsplitters, mirrors, and Iodine vapor cell, to the nonuniformity of the pixel distribution and element orientation in the cameras. Although these obstacles may not be insurmountable, the anticipated difficulties involved in a hardware solution led to the use of image processing techniques to improve image alignment, as described by Meyers (9).

The method implemented in software was based on determining the set of linear equations needed to move the centroid position of each grid point on a calibration card from its imaged position to its ideal position. This image warping procedure produced image alignment to subpixel accuracy over the entire normalized image. Since interlaced cameras were used, each acquired field (the odd rows (field 1) were acquired during the first $1 / 60$ th of a second, while the image acquired previously by the even rows (field 2) was transferred out; during the next $1 / 60$ th of a second the process switched) was processed as a separate image to maintain simultaneous data acquisition. Variations in sensitivity among the pixels in each CCD camera were also removed using standard flat field calibration procedures. The acquired signal and reference images were processed again using these techniques to improve the measurements. An example of the reprocessed data is the cross flow measurements illustrated in Fig 8.

Combining the contours of velocity obtained by the laser velocimeter, Fig 7, with the DGV images, Fig 8, provides a direct comparison of the two measurement techniques, Fig 9. Measurements of the left vortex yield the expected profiles for stable vortical flow. The laser velocimetry measurements of the right vortex also yielded the expected profiles, but the DGV images showed profiles that were representative of solid bodies of revolution. Laser light sheet visualization of this flow, Fig 10, indicates that the left vortex remained stable over the model while the right vortex burst. This phenomena was traced to a yaw of 0.2 degrees in the mounting of the model. 

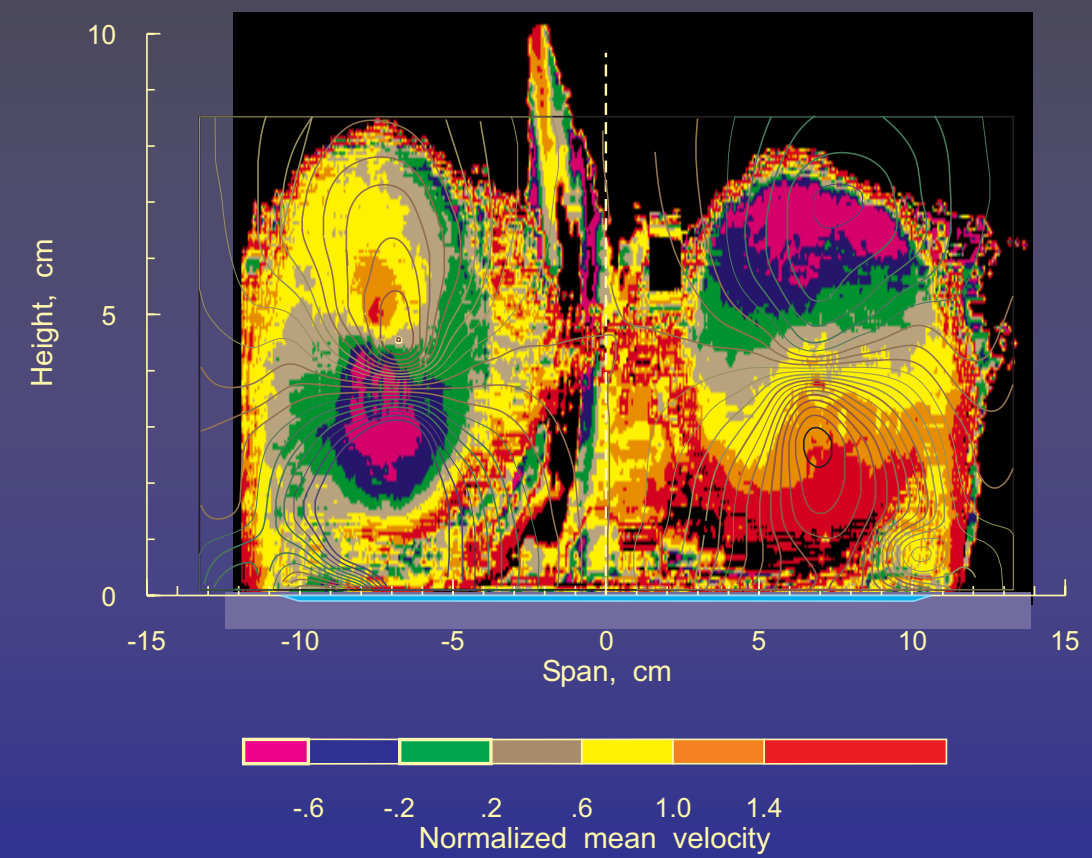

Fig 9. Comparison of LV and DGV measurements of the velocity field for the component along the direction 71.5 degrees from streamwise in the horizontal plane.

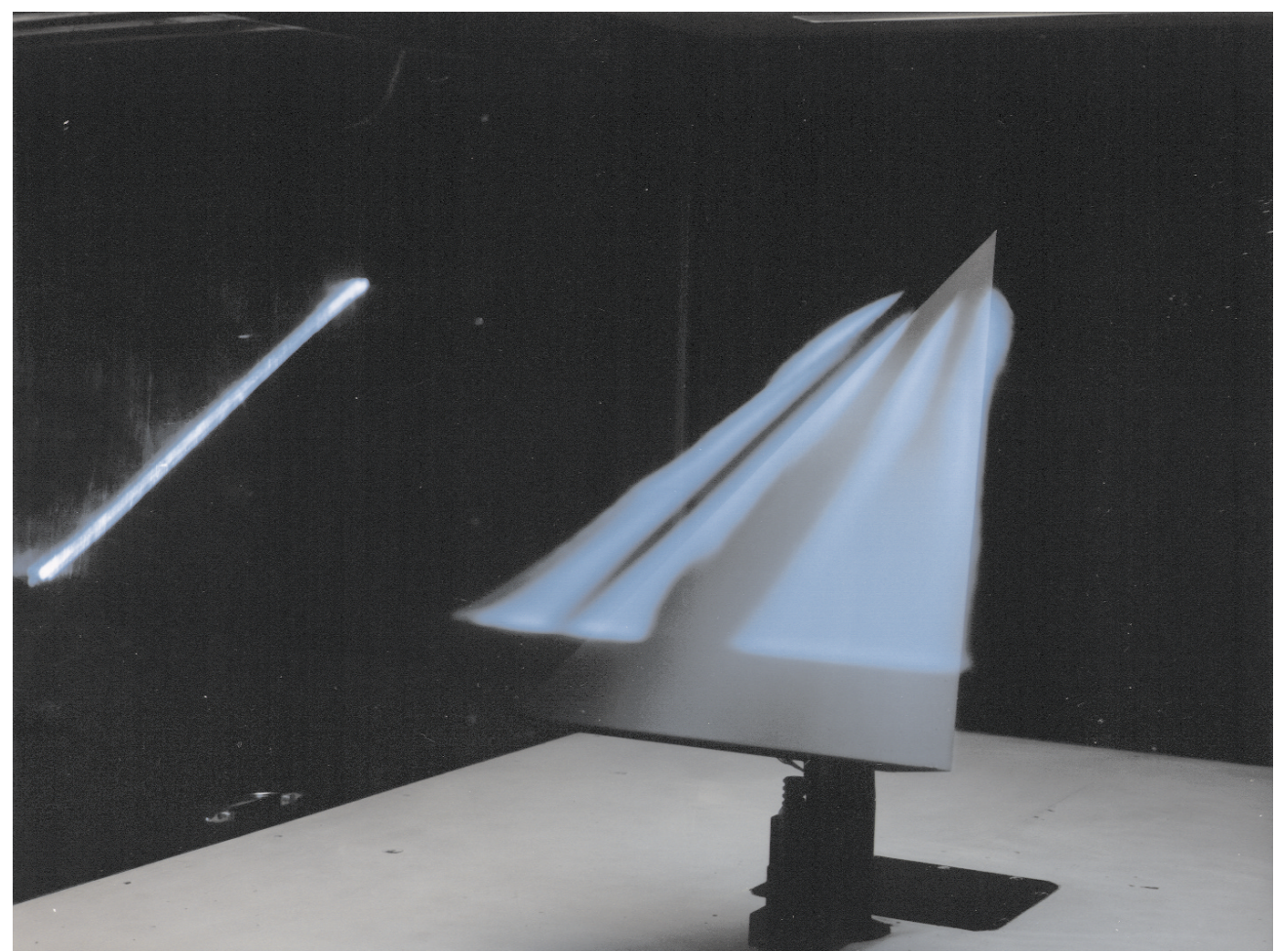

Fig 10. Laser light sheet visualization of the vortical flow above a 75-degree delta wing. 
Verification that the DGV would actually yield the striped pattern of velocity contours from a solid body of revolution could be obtained by measuring the horizontal velocity component of a rotating wheel. The DGV was configured to yield measurements of the horizontal velocity component, 39 degrees out of plane from the wheel as indicated in Fig 11. The resulting measurements, presented in Fig 12, clearly show the stripped pattern. The measurement accuracy of the DGV using the outlined data processing techniques can be illustrated by the results along the vertical diameter of the rotating wheel, Fig 13 . The 307 velocity measurements comprising the velocity profile along the vertical diameter of the $13.34 \mathrm{~cm}$ wheel have an average error of $-0.69 \mathrm{~m} / \mathrm{sec}$ and a standard deviation of $3.61 \mathrm{~m} / \mathrm{sec}$ over the velocity range from $-65 \mathrm{~m} / \mathrm{sec}$ to $65 \mathrm{~m} / \mathrm{sec}$.

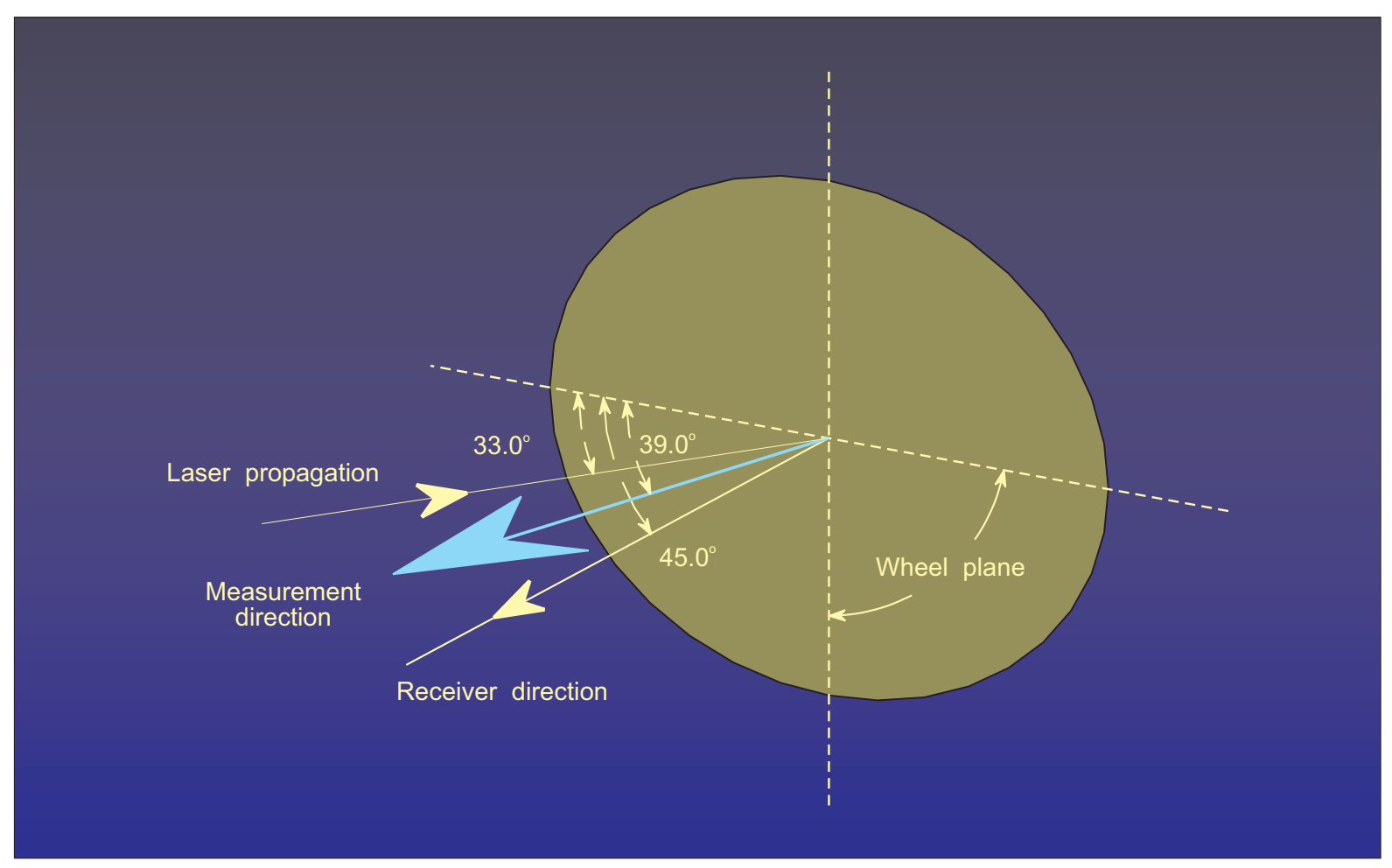

Fig 11. DGV measurement direction for the wheel experiment. 


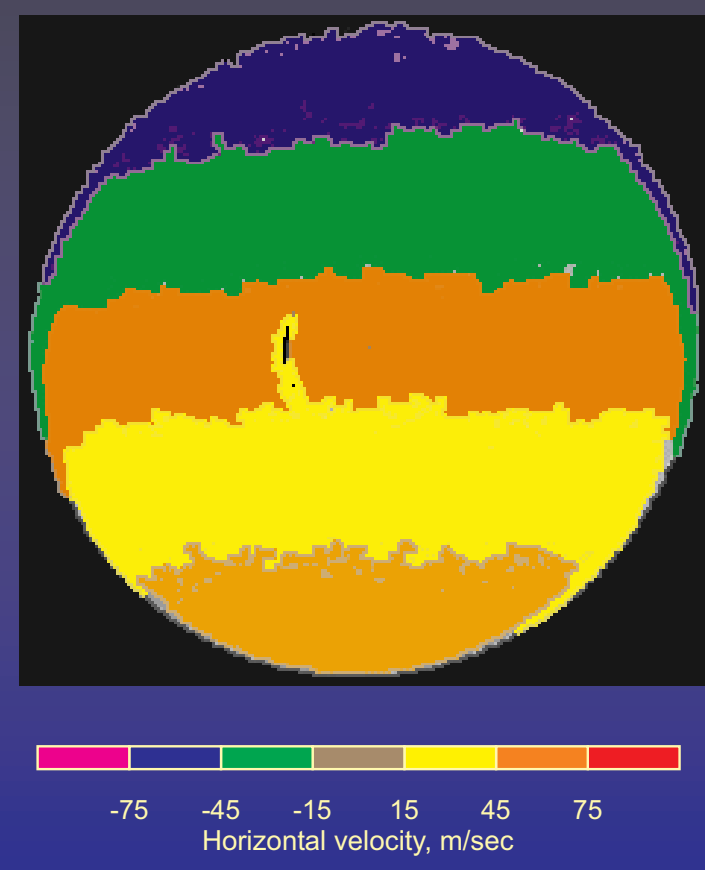

Fig 12. DGV measurements of the horizontal velocity field (average of 5 frames) of a wheel rotating at $12,000 \mathrm{rpm}$.

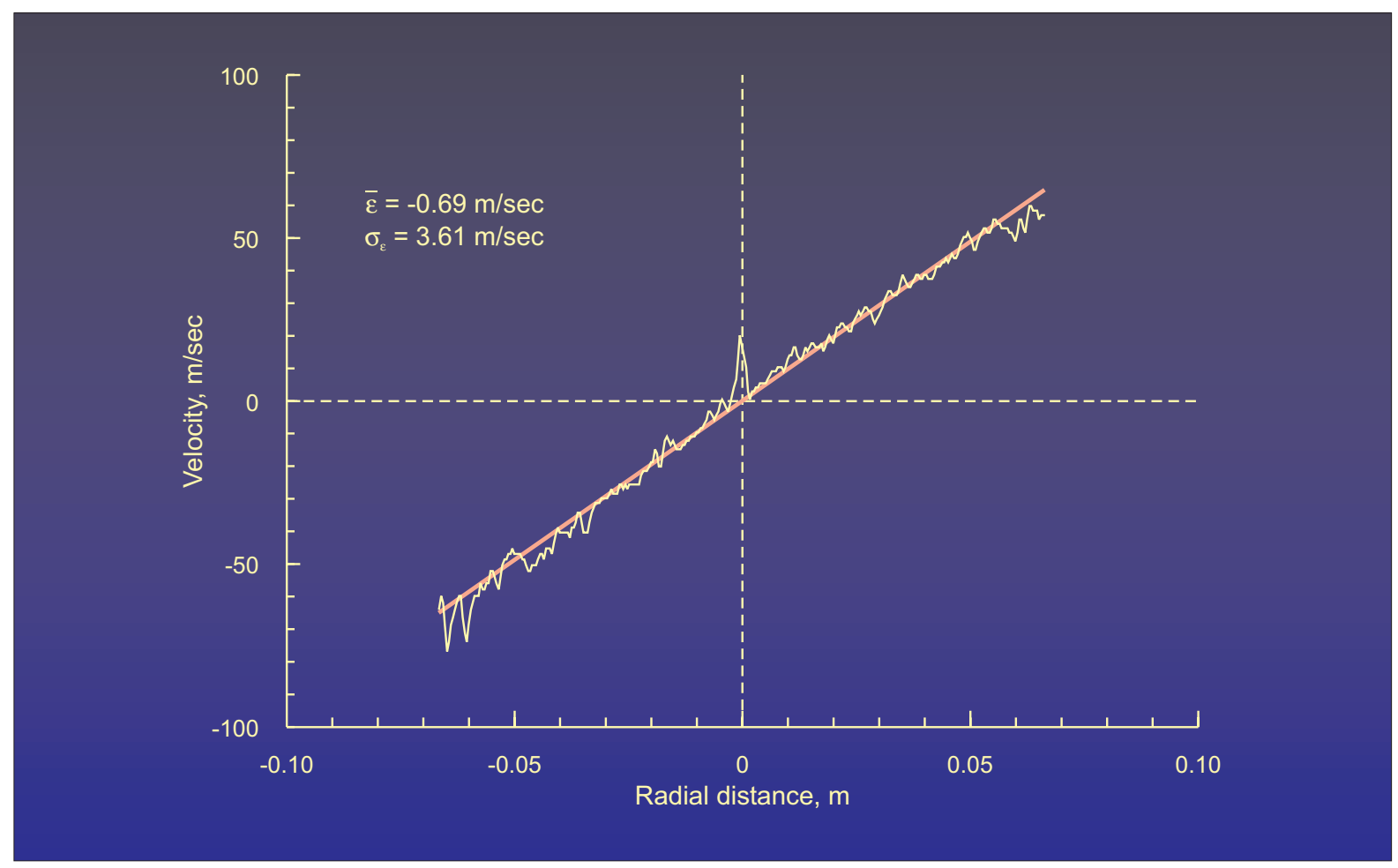

Fig 13. DGV measurements of the horizontal velocity along the vertical diameter of a wheel rotating at $12,000 \mathrm{rpm}$. 


\section{SUMMARY}

A new measurement technique, Doppler global velocimetry, has been described along with results from an experimental investigation of a vortical flow field. The results of this investigation, when compared to conventional laser velocimeter measurements, indicate that this technique is capable of describing the global velocity field within a measurement plane in real time.

\section{REFERENCES}

1. Komine, H.; Brosnan, S. J.; Litton, A. B.; and Stappaerts, E. A.: Real Time, Doppler Global Velocimetry. AIAA-91-0337, January 1991.

2. Yeh, Y.; and Cummins, H. Z.: Localized Fluid Flow Measurements with a He-Ne Laser Spectrometer. Applied Physics Letters, vol. 4, no. 10, pp. 176-178, May 1964.

3. Meyers, J. F.; and Komine, H.: Doppler Global Velocimetry - A New Way to Look at Velocity. Proceedings of the ASME 4th International Conference on Laser Anemometry, Advances and Applications, Cleveland, OH, vol. 1, pp. 289-296, August 5-9, 1991.

4. Meyers, J. F.; Lee, J. W.; and Cavone, A. A.: Signal Processing Schemes for Doppler Global Velocimetry. Proceedings of the IEEE - 14th International Congress on Instrumentation in Aerospace Simulation Facilities, Rockville, MD, pp. 321-328, October 27-31, 1991.

5. Usry, J. W.; Meyers, J. F.; and Miller, L. S.: Doppler Global Velocimeter Measurements of the Vortical Flow Above a Thin Delta Wing. AIAA-92-0005, January 1992.

6. Sellers, W. L., III; and Kjelgaard, S. O.: The Basic Aerodynamics Research Tunnel-A Facility Dedicated to Code Validation. AIAA88-1997, May 1988.

7. Meyers, J. F.: A Three Dimensional View of Velocity Using Lasers. Proceedings of the 10th International Invitational Symposium on Unification of Finite Element Methods in Theory and Test, Worcester, MA, pp. 431-458, July 18-19, 1991. 
8. Meyers, J. F.; and Hepner, T. E.: Measurements of Leading Edge Vortices from a Delta Wing Using a Three Component Laser Velocimeter. AIAA-88-2024, May 1988.

9. Meyers, J. F.: Doppler Global Velocimetry - The Next Generation? AIAA 17th Aerospace Ground Testing Conference, Nashville, TN, paper no. AIAA-92-3897, July 6-8, 1992. 